\title{
PAIR CORRELATIONS AND RANDOM WALKS ON THE INTEGERS
}

\author{
RADHAKRISHNAN NAIR - ENTESAR NASR \\ Dedicated to the memory of Professor Pierre Liardet.
}

\begin{abstract}
The paper gives conditions for a sequence of fractional parts of real numbers $\left(\left\{a_{n} x\right\}\right)_{n=1}^{\infty}$ to satisfy a pair correlation estimate. Here $x$ is a fixed nonzero real number and $\left(a_{n}\right)_{n=1}^{\infty}$ is a random walk on the integers.
\end{abstract}

\section{Communicated by Oto Strauch}

Let $X$ be a $\mathbb{Z}$-valued function defined on the probability space $(\Omega, \beta, P)$ with characteristic function $\phi(\xi)=\mathbb{E}\left(e^{i X(.) \xi}\right)$ and let $\chi=\left\{X_{n}: n \geq 1\right\}$ be a sequence of independent copies of $X$. For a positive integer $n>0$ let $a_{n}=X_{1}+\cdots+X_{n}$ and let $a_{0} \equiv 0$. The sequence of integers $\left(a_{n}\right)_{n \geq 1}$ is the random walk which we assume to satisfy $|\phi(t)-1| \geq C|t|$, some $C>0$. This last property follows, for instance, if the random walk and its absolute value have finite non-zero mean [Sp, p. 62]. In W1] the condition $|\phi(t)-1| \geq C|t|$ is said to follow from the assumption that the random is aperiodic and transient - a claim the authors was unable to verify. This is then used to deduce a discrepancy estimate for the sequence $\left(X_{n}(x)\right)_{n=1}^{\infty}$. This is so, for instance (as a consequence of the law of large numbers) if $\mathbb{E}|X|<\infty$ and $\mathbb{E} X \neq 0$ or if $X$ is centred and $\frac{a_{n}}{n \frac{1}{\alpha}}$ converges in distribution to $F_{\alpha}$, a stable law of index $\alpha \in(0,1)$. This second class of examples can be deduced using a local limit theorem of Stone $\mathrm{St}$. For a real number $x$ let $X_{n}(x)=a_{n} x$. For an interval $I$ let $\chi_{I}(x)$ denote the characteristic function of the set $I$. This means that we have

$$
\chi_{I}(x)=1 \quad \text { if } x \in I \quad \text { and } \quad \chi_{I}(x)=0 \text { otherwise. }
$$

2010 Mathematics Subject Classification: 11K38, 60 G50.

Keywords: pair correlation, random walks. 
For a real number $y$ let $\{y\}$ denote its fractional part. Set

and then define

$$
V_{N}(I)(x)=\sum_{1 \leq n<m \leq N} \chi_{I}\left(\left\{X_{n}(x)-X_{m}(x)\right\}\right)
$$

$$
\Delta_{N}(x)=\sup _{I \subseteq \mathbb{T}}\left|V_{N}(I)-\frac{N(N-1)}{2} \operatorname{leb}(I)\right|,
$$

where the supremum is over all intervals $I$ in the one dimensional torus $\mathbb{T}$. Let $\|x\|=\min _{n \in \mathbb{Z}}|x-n|=\min (\{x\},\{1-x\})$. Let $\eta$ be a positive real number or infinity. The irrational number $x$ is said to be of type $\eta$ if $\eta$ is the supremum of all $\gamma$ for which liminf $\operatorname{in}_{q \rightarrow \infty} q^{\gamma}\|q x\|=0$. Using Dirichlet's theorem on diophantine approximation we can deduce for all irrational $x$ that $\lim _{\inf _{q \rightarrow \infty}} q^{\gamma}\|q x\|=0$ so $\eta \geq 1$. On the other hand, the Thue-Siegel-Roth theorem tells us that for every irrational algebraic $x$ and every $\epsilon>0$ there exists a constant $c(x, \epsilon)>0$ such that

$$
\left|x-\frac{p}{q}\right| \geq \frac{c(x, \epsilon)}{q^{2+\epsilon}}
$$

holds for all coprime integers $q>0$ and $p$, so that algebraic $\eta$ must be of type 1 . Liouville numbers can easily be used to show constructively that there exist real numbers that of type strictly greater than 1 .

Our theorem is the following.

Theorem. Suppose $\left(X_{n}(x)\right)_{n=1}^{\infty}$ is as described above that $x$ has type $\eta>1$. Then given $\epsilon>0$,

$$
\Delta_{N}(x)=o\left(N^{2-\frac{1}{\eta}+\epsilon}\right)
$$

for $P$ almost all $\omega \in \Omega$.

Let $D_{N}(x)$ denote the $N$-term discrepancy of the sequence $\left(X_{n}(x)\right)_{n \geq 1}$. See [KN, p 88] for the definition. M. Weber [W2, p 411] has given an estimate for almost everywhere behaviour of $D_{N}(x)$ as $N$ tends to infinity in terms of the type of $x$ and the properties of the the function $\phi$. The formulation is however somewhat involved and forgone here. Results like our theorem, where $\left(a_{n}\right)_{n=1}^{\infty}$ is fixed and deterministic and $x$ is random are now known. See [NP] for details and further background.

We proceed by a series of lemmas. For real $x$ let $e(x)=e^{2 \pi i x}$ and let

$$
\theta_{N}(h)=\sum_{n=1}^{N} e\left(h a_{n} x\right) \quad(N=1,2, \cdots) .
$$

We need the following lemma taken for W1. 
Lemma 1. For integers $N \geq R \geq 1$ one has

$$
\mathbb{E}\left|\theta_{N}(m)-\theta_{R}(m)\right|^{2} \leq \min \left(\frac{7(N-R)}{|\phi(m x)-1|}, N-R\right) .
$$

Let $\left(Y_{t}\right)_{t=1}^{\infty}$ be a sequence of measurable functions defined on a measure space $\Omega$ and then write

$$
S_{j}=\sum_{1 \leq t \leq j} Y_{t}, \quad \text { for } j=1,2, \cdots
$$

We can define

$$
Y_{r s}=\sum_{r \leq t<s} Y_{t}\left(=S_{s}-S_{r}\right), \quad \text { for } r<s
$$

and let

$$
M_{n}=\sup _{1 \leq j \leq n}\left|S_{j}\right|
$$

We have the following elementary lemma proved in $\mathrm{NP}$.

LEMMA 2. For $K \geq 1$,

$$
\int_{\Omega} M_{2^{K}}^{2}(\omega) \mathrm{d} \omega \leq(K+1)\left(\sum_{i=1}^{K+1} \sum_{\nu=1}^{2^{i}-1} \int_{\Omega}\left|Y_{\nu 2^{(K+1)-i},(\nu+1) 2^{(K+1)-i}}\right|^{2}(\omega) \mathrm{d} \omega\right) .
$$

Thus if $K=1,2, \cdots$

$$
\begin{aligned}
\mathbb{E}\left|\max _{1 \leq j \leq 2^{K}} \theta_{j}(m)\right|^{2} & \leq(K+1)\left(\sum_{i=1}^{K+1} \sum_{\nu=1}^{2^{i}-1} \mathbb{E} \mid \theta_{\nu 2^{(K+1)-i}}(m)-\theta_{\left.(\nu+1) 2^{(K+1)-i}(m)\right|^{2}}\right) \\
& \leq(K+1)\left(\sum_{i=1}^{K+1} \sum_{\nu=1}^{2^{i}-1}\left(\frac{7.2^{(K+1)-i}}{|\phi(m x)-1|}\right)\right) \\
& \leq(K+1)\left(\sum_{i=1}^{K+1} 2^{i-1}\left(\frac{7.2^{(K+1)-i}}{|\phi(m x)-1|}\right)\right) \\
& \leq \frac{7}{2}(K+1)^{2}\left(\frac{2^{(K+1)}}{|\phi(m x)-1|}\right) .
\end{aligned}
$$

Thus, using the Erdös-Turan inequality [KN, p 112-4], we can show that for $L \geq 1$, there exists $C>0$, 


$$
\begin{aligned}
\mathbb{E}\left|\max _{1 \leq j \leq 2^{K}} \Delta_{j}(x)\right| & \leq C\left(\frac{2^{2(K+1)}}{L}+\sum_{h=1}^{L} \frac{1}{h}\left(2^{K+1}+\mathbb{E} \max _{1 \leq j \leq 2^{K}}\left|\theta_{j}(h)\right|^{2}\right)\right) \\
& \leq C\left(\frac{2^{2(K+1)}}{L}+\sum_{h=1}^{L} \frac{1}{h}\left(2^{K+1}+\frac{7}{2}(K+1)^{2}\left(\frac{2^{(K+1)}}{|\phi(h x)-1|}\right)\right)\right) .
\end{aligned}
$$

Let $\Lambda: \mathbb{R}_{+} \rightarrow \mathbb{R}_{+}$be a non-decreasing and such that for any $m \in \mathbb{N}$,

$$
\sum_{h=1}^{L} \frac{1}{h|\phi(h x)-1|} \leq \Lambda(L) .
$$

Then the left hand side of (1)

$$
\ll\left(\frac{2^{2(K+1))}}{L}+(\log L) 2^{(K+1)}+\Lambda(L)(K+1)^{2} 2^{(K+1)}\right) .
$$

Here, of course, « denotes Vinogradov order notation. Recall that there exists

$$
C>0 \text { such that }|1-\phi(t)| \geq C|t| .
$$

Since

we therefore have

$$
\phi(h x)=\phi(\{h x\}),
$$

$$
\sum_{h=1}^{L} \frac{1}{h|\phi(h x)-1|}=O\left(\sum_{h=1}^{L} \frac{1}{h \| h x \mid}\right) .
$$

If $x$ is irrational of type $\eta>1[\mathrm{KN}$, p. 123, Lemma 3.3] for any $\epsilon>0$, then

$$
\sum_{h=1}^{L} \frac{1}{h\|h x\|}=O\left(L^{\eta-1+\epsilon}\right) .
$$

In consequence, we can choose $\Lambda(L)=L^{\eta-1+\epsilon}$ the right hand side of (2) is

$$
\ll \frac{2^{2(K+1)}}{L}+(\log L) 2^{(K+1)}+\Lambda(L)(K+1)^{2} 2^{(K+1)} .
$$

Choosing $L$ essentially optimally $2^{K} \approx L \Lambda(L)=L^{\eta+\epsilon}$ the right hand side of (3) is

$$
\ll 2^{(K+1)\left(2-\frac{1}{\eta}+\epsilon\right)}(K+1)^{2} .
$$

Now we will complete the proof of our Theorem . 
Proof of Theorem. Given $\epsilon, \epsilon_{0}>0$, we define

$$
E_{\epsilon, \epsilon_{0}}=\left\{\omega \in \Omega: \Delta_{N}(\omega, x)>N^{2-\frac{1}{\eta}+\epsilon}(\log N)^{3+\epsilon_{0}} \text { for infinitely many } N\right\} .
$$

We now proceed to show the $P$ measure of $E_{\epsilon, \epsilon_{0}}$ is zero. If we denote

$$
A_{K}=\left\{\omega \in \Omega: \max _{2^{K-1} \leq m<2^{K}} \Delta_{m}(\omega, x)>2^{K\left(2-\frac{1}{\eta}+\epsilon\right)} K^{3+\epsilon_{0}}\right\} \text { for each } K \geq 1,
$$

then one easily sees that

$$
E_{\epsilon, \epsilon_{0}} \subseteq \bigcap_{r=1}^{\infty} \bigcup_{K=r}^{\infty} A_{K}
$$

Using (4) we can bound

$$
\begin{aligned}
\mathrm{P}\left(A_{K}\right) & \leq \frac{\mathbb{E} \mid \max _{2^{K-1} \leq m<2^{K} \Delta_{m}(x) \mid}^{2^{K\left(2-\frac{1}{\eta}+\epsilon\right)} K^{3+\epsilon_{0}}}}{} \\
& \leq \frac{C 2^{K\left(2-\frac{1}{\eta}+\epsilon\right)} K^{2}}{K^{3+\epsilon_{0}} 2^{K\left(2-\frac{1}{\eta}+\epsilon\right)}} \\
& \leq C K^{-\left(1+\epsilon_{0}\right)} \quad \text { for sufficiently large } C>0 .
\end{aligned}
$$

In particular, now we can observe that

$$
\sum_{K=1}^{\infty} \mathrm{P}\left(A_{K}\right) \leq \sum_{K=1}^{\infty} K^{-\left(1+\epsilon_{0}\right)}<+\infty .
$$

It follows from the Borel-Cantelli lemma that $\mathrm{P}\left(E_{\epsilon, \epsilon_{0}}\right)=0$.

ACKnowledgement. We thank the referee for comments which materially improved the presentation of this paper.

\section{REFERENCES}

[KN] KUIPERS, L.-NIEDERREITER, H.: Uniform Distribution of Sequences. Pure and Applied Mathematics, Wiley-Interscience [John Wiley \& Sons], New York-London-Sydney, 1974.

[NP] NAIR, R.-POLLICOTT, M.: On pair correlations of sequences in higher dimensions, Israel J. Math. 157 (2007), no. 1, 219-238.

[Sp] SPITZER, F. L.: Principles of Random Walks. Second ed. Graduate Texts in Mathematics, Vol. 34. Springer-Verlag, New York-Heidelberg, 1976. 


\section{RADHAKRISHNAN NAIR - ENTESAR NASR}

[St] STONE, C. T.: On local and ratio limit theorems, in: Proc. of the Fifth Berkeley Symp. on Math. Statist. and Probab. Vol. 2: Contributions to Probability Theory, Part 2, (L. M. Le Cam and J. Neyman, Eds.), University of California Press, Berkeley, California, 1967, pp. 217-224

[W1] WEBER, M.: Discrepancy of randomly sampled sequences of reals, Math. Nachr. 271 (2004), 105-110.

[W2] WEBER, M.: Dynamical Systems and Processes. IRMA Lectures in Mathematics and Theor. Physics Vol 14, European Mathematical Society (EMS), Zürich, 2009.

Received March 10, 2015

Accepted March 22, 2016

\section{Radhakrishnan Nair Entesar Nasr}

Department of Mathematical Sciences

University of Liverpool

Mathematical Sciences Building

Liverpool L69 7ZL

UNITED KINGDOM

E-mail: nair@liverpool.ac.uk e.m.nasr@liverpool.ac.uk 